\title{
Cell-based immunotherapy in stage IIIA inflammatory breast cancer with declining innate immunity following successive chemotherapies: A case report
}

\author{
RANGANATHAN CHIDAMBARAM ${ }^{1,2}$, HIROSHI TERUNUMA ${ }^{3}$, MADASAMY BALAMURUGAN ${ }^{1,4}$, \\ VIDYASAGAR DEVAPRASAD DEDEEPIYA ${ }^{5}$, PREMKUMAR SUMANA ${ }^{6,7}$, RAJAPPA SENTHILKUMAR ${ }^{8}$, \\ MATHAIYAN RAJMOHAN $^{8}$, RAMALINGAM KARTHICK ${ }^{8}$, SENTHILKUMAR PREETHY $^{8,9}$ \\ and SAMUEL J.K. ABRAHAM ${ }^{5,10}$
}

${ }^{1}$ RUMA Biotherapy and Research Centre, Moolakulam, Puducherry 605010; ${ }^{2}$ Department of Radiology, Sri Lakshmi Narayana Institute of Medical Sciences, Bharath University, Chennai, Tamil Nadu 600073, India; ${ }^{3}$ Biotherapy Institute of Japan, Tokyo 135-0051, Japan; ${ }^{4}$ Department of Pathology, Tagore Medical College and Hospital, Chennai, Tamil Nadu 600127;

${ }^{5}$ The Mary-Yoshio Translational Hexagon (MYTH), Nichi-In Centre for Regenerative Medicine (NCRM), Chennai, Tamil Nadu 600034; ${ }^{6}$ Chennai Meenakshi Multispeciality Hospital Limited, Chennai, Tamil Nadu 600004;

${ }^{7}$ Dr Kamakshi Memorial Hospital, Chennai, Tamil Nadu 600100; ${ }^{8}$ The Fujio-Eiji Academic Terrain (FEAT),

Nichi-In Centre for Regenerative Medicine (NCRM), Chennai, Tamil Nadu 600034; ${ }^{9}$ Hope Foundation (Trust), Chennai, Tamil Nadu 600094, India; ${ }^{10}$ Department of Surgery, Yamanashi University School of Medicine, Chuo, Yamanashi 409-3898, Japan

Received January 11, 2017; Accepted July 5, 2017

DOI: $10.3892 /$ mco.2017.1333

\begin{abstract}
Cancer stem cells in breast cancer migrating to the bone marrow may cause future metastasis, particularly during periods of decreased immunity. Natural killer (NK) cells have a role in immune surveillance and are able to target cancer stem cells. The present study reported a case in which NK cell-based autologous immune enhancement therapy was used combined with conventional treatments in a patient with stage IIIA breast cancer, yielding $>28$ months of disease-free survival. However, there was a gradual decline in the in vitro expansion of NK cells with subsequent chemotherapeutic treatments. As this NK cell decline following chemotherapy may contribute to cancer cell immune evasion and future metastasis; modifying current cancer therapies in order to
\end{abstract}

Correspondence to: Dr Samuel J.K. Abraham, The Mary-Yoshio Translational Hexagon (MYTH), Nichi-In Centre for Regenerative Medicine (NCRM), C-30, 3rd floor, LICET, Loyola College Campus, Mahalingam Street, Mahalingapuram, Nungambakkam, PB 1262, Chennai, Tamil Nadu 600034, India

E-mail: drsam@nichimail.jp

Abbreviations: AIET, autologous immune enhancement therapy; NK cells, natural killer cells; PET-CT, positron emission tomography-computed tomography; ER, estrogen receptor; PR, progesterone receptor; EGFR, epidermal growth factor receptor

Key words: breast cancer, autologous immune enhancement therapy, natural killer cells, cancer stem cells, immune loopholes avoid potentially compromising the immune system may lead to improved treatment outcomes.

\section{Introduction}

Breast cancer is the most common invasive cancer in females and the data indicate that this cancer led to 458,503 deaths worldwide in 2008 (1). As a number of studies are ongoing for identifying therapeutic strategies to combat this form of cancer, the progress is slow owing to its complexity. The resistance of breast cancer stem cells to conventional therapies such as chemotherapy (2) increases this complexity, as these cells are responsible for recurrence and disease progression. It has been reported that, even at the early stages of the disease, a portion of breast cancer cells may migrate to the bone marrow, and their entry into the bone marrow is facilitated by mesenchymal stem cells (3). These migrated cancer cells remain dormant, becoming active later thereby causing recurrence or advancement of the disease. $\mathrm{CD} 3{ }^{-} \mathrm{CD} 56^{+}$or $\mathrm{CD} 16^{+}$ natural killer (NK) cells that possess the ability to identify and kill target cancer cells (4), particularly cancer stem cells $(5,6)$ without prior sensitization makes them an important player in immunosurveillance and cancer defense in vivo. Therefore, NK cell-based immunotherapy may be a promising therapeutic strategy against cancer (4). There have been several studies investigating the role of NK cells in anticancer immunity and the outcome of cell-based immunotherapy in various types of solid cancer (4,7-9). In breast cancer, there have been reports on positive outcomes following cell-based immunotherapy $(10,11)$. Chemotherapy has been reported to cause a decline in NK cell number and function (12), which 
may compromise their use in immunotherapy. In this context, the current study reports on the use of NK cell-based autologous immune enhancement therapy (AIET) in a patient with stage IIIA breast cancer, in whom a positive outcome was observed after adding AIET to conventional treatments. The observations concerning the decrease in the NK cell in vitro growth expansion with subsequent chemotherapy cycles in the patient are also reported.

\section{Case report}

Presenting complaints. In October 2012, a 29-year-old Indian female presented to the Chennai Meenakshi Multispeciality Hospital Limited (Chennai, India), reporting a history of pain and a lump in the left breast since August 2012. The patient had given birth to a child in July 2012.

Clinical findings. Following preliminary investigations, a Tru-Cut biopsy in October 2012 revealed infiltrating lobular carcinoma of the left breast. The cancer was aggressive and inflammatory in nature, with rapidly progressing breast tenderness, pain and erythema. Whole-body positron emission tomography-computed tomography (PET-CT) scanning revealed a metabolically active large lobulated heterogeneously enhancing lesion $(12.4 \times 7.4 \mathrm{~cm})$ (Fig. 1A), involving all quadrants of the left breast with diffuse left breast skin thickening and multiple discrete satellite nodules $(<15 \mathrm{~mm})$ surrounding the mass lesion and metabolically active multiple left axillary, pectoral and left upper internal mammary lymph nodes (Fig. 1A). Immunohistochemistry revealed histological characteristics compatible with infiltrating ductal carcinoma of the breast (Bloom \& Richardson's provisional Grade 8) (13) ER-positive, PR-negative, Her2/Neu-negative, Ki-67-positive (86\%) and EGFR- and cytokeratin 5-negative. The patient underwent three cycles of preoperative chemotherapy (between October 2012 and December 2012) with doxorubicin, docetaxel and cyclophosphamide, followed by left modified radical mastectomy (December 2012) followed by three cycles of postoperative chemotherapy (between January and February 2013). The histopathological examination following the surgery and chemotherapy established the tumor to be pT3 N2a Mx stage IIIA (14).

Therapeutic focus and assessment. The patient simultaneously underwent 12 transfusions of NK cell-based AIET (15-17) between November 2012 and February 2013 planned in accordance with the chemotherapy cycles. Approximately 200-210 ml of peripheral blood (PB) was withdrawn for the first 3 cycles ( 3 transfusions in 1 cycle, 9 transfusions in total) and then for the 10th transfusion, only $40 \mathrm{ml}$ of PB was withdrawn as the patient's general health condition was low. A quantity of $185 \mathrm{ml}$ of PB was withdrawn for the 11 and 12th transfusions. For each AIET transfusion, the NK cells isolated from peripheral blood mononuclear cells were culture-expanded in vitro, employing autologous plasma without using feeder layers or animal or allogeneic serum, based on previously described protocols $(11,15-17)$ for 10-12 days before being infused into the patient. The patient also underwent 5,400 cGy in 27 fractions of radiotherapy between March 2013 and April 2013.
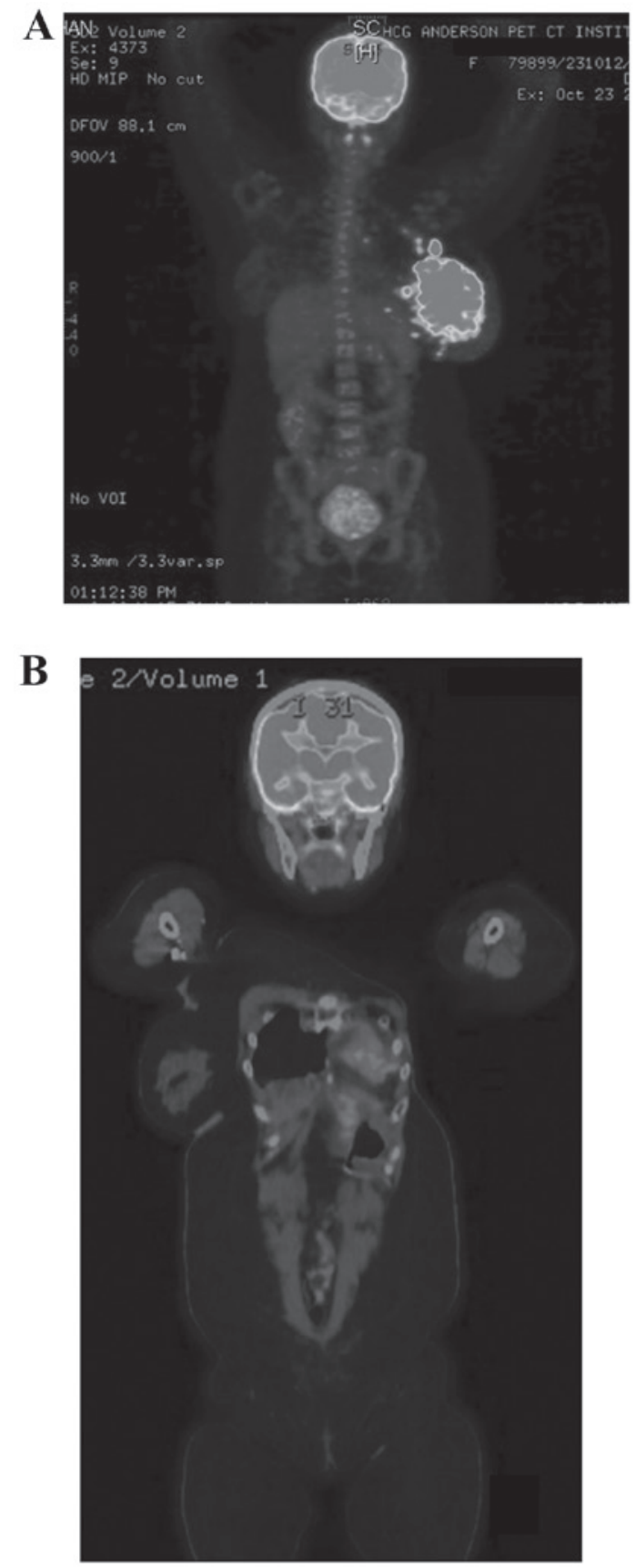

Figure 1. Whole-body positron emission tomography-computed tomography. (A) Image taken prior to the start of the treatments. (B) Follow-up scan image in March 2015, showing no evidence of mass lesions or enlarged lymph nodes.

Follow-up and outcomes. Fig. 2A presents the total number of leukocytes, which remained close to normal throughout the therapy. Fig. 2B shows the declining NK cell counts following their in vitro expansion, parallel to subsequent chemotherapy cycles, and the decrease became more profound after the surgery. There were no adverse reactions following the AIET. Despite the decreased NK cells counts after in vitro expansion, there was a subjective improvement in the quality of life after AIET and the patient reported improved tolerance to the side effects of the chemotherapy, possibly as AIET was administered concurrently. The patient has been disease-free for $>28$ months and a PET-CT scan in February 2014 identified no evidence of recurrence. Follow-up scans in June 2014 and March 2015 
A

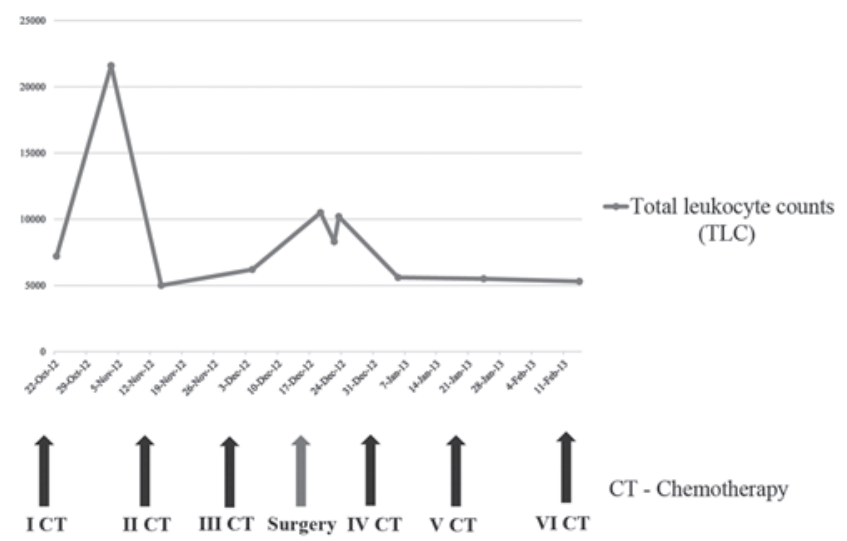

B

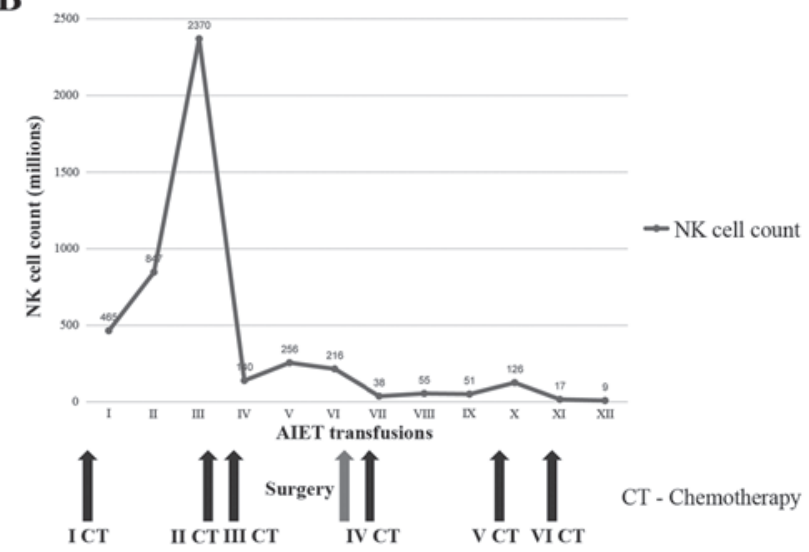

Figure 2. (A) Total leukocyte counts over the course of the treatment. (B) Decrease observed in NK cell counts following in vitro expansion with progressive cycles of chemotherapy. NK, natural killer; AIET, autologous immune enhancement therapy.

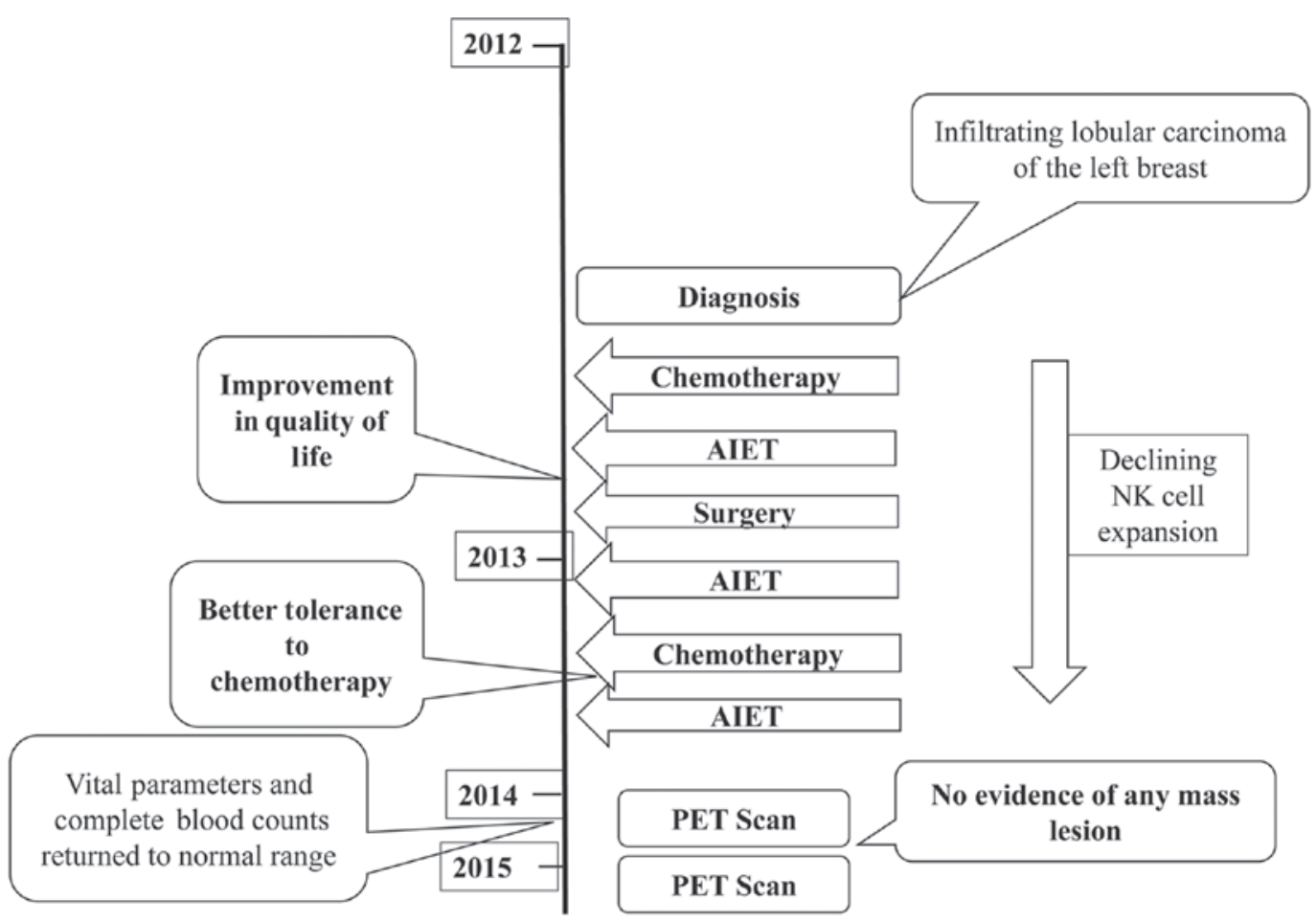

Figure 3. Timeline of interventions and outcomes. NK, natural killer; AIET, autologous immune enhancement therapy; PET, positron emission tomography,

(Fig. 1B) also reported that there was no evidence of any mass lesion or large lymph nodes in the abdomen or the pelvis. Fig. 3 depicts the timeline of the interventions and outcomes.

Informed consent and ethics policy. Verbal and written informed consent was obtained from the patient for the inclusion of her medical and treatment history within this case report. The study was performed in accordance with the ethical standards laid down in the 1964 Declaration of Helsinki and all subsequent revisions.

\section{Discussion}

In a study that examined the prognosis of inflammatory breast cancer (IBC) by screening nearly 3,650 patients with breast cancer, the median progression-free survival period was 17 months. This study was from the All India Institute of Medical Sciences (AIIMS), a large tertiary care center in India (18) and this may be considered as representative of Indian data on IBC, which is relevant to the present case, as the patient described is of Indian ethnicity. The patients in the AIIMS study underwent conventional therapies such as chemotherapy, modified radical mastectomy, radiotherapy and hormonal therapy as indicated. The patient described in the present case is having a disease-free survival period of $>28$ months following a combination therapy of chemotherapy, radiotherapy and surgery along with AIET, and is continuously under follow-up. The longer disease-free survival period may be attributable to the combination of AIET with the conventional therapies, which requires further validation in larger number of patients. 
The complex nature of breast cancer biology, due to its genetic and hormonal influence, is further complicated by the migration of its cells to bone marrow even at an early stage (3), which leads to later recurrence of the disease. More complex is IBC, which has a very aggressive presentation, with the majority of patients ultimately succumbing to the disease. The present study examined in vitro NK cell expansion during administration of cell-based AIET concurrently with chemotherapy and surgery in a patient with stage IIIA IBC. The observation from the data is that, in this patient, the in vitro expansion of NK cells from PB withdrawn prior to the start of the chemotherapy cycles is higher (I-III transfusions), whereas with progressive chemotherapy, the quantity of NK cells following in vitro expansion decreased. In particular, after the surgery and the fourth cycle of chemotherapy, the expansion of NK cells decreased markedly (Fig. 2B). There are mixed reports in the literature on the effects of chemotherapy on NK cells. Although certain reports suggest that chemotherapy reduces the count and cytotoxicity of NK cells $(12,19)$, another suggested an increase in NK cell cytotoxicity following chemotherapy (20). The present case report suggests that there is decrease in the in vitro expansion of NK cells with subsequent chemotherapy cycles. Although earlier studies have discussed the effects of chemotherapy on either the count or the in vitro cytotoxicity of the NK cells $(12,19,20)$, to the best of our knowledge the current report is the first to provide details on the in vitro expansion potential of the NK cells, which has clinical significance as these expanded cells were used for immunotherapy in the patient described herein. Tai et al (21) reported that surgical stress induces dysfunction of NK cells, impairing their cytotoxic ability, thereby promoting tumor metastasis. The present report is in line with the Tai et al study, in which, following surgery, there was a significant decrease in the quantity of NK cells after in vitro expansion. Their cytotoxicity may also have been affected, which requires further study. The present report is of interest not only for the development of more focused therapies, but also for developing targeted therapies that will not compromise the cells of the immune system, as high densities of cytotoxic immune cells have been correlated with good prognosis in cancer (22-24), and proper functioning of immune cells such as NK cells is necessary to prevent metastasis (21). However, despite reduced NK cell quantities after in vitro expansion, the patient in the present case has remained disease-free for $>28$ months, which suggests that AIET, even with compromised NK cell function, may be able to contribute to a favorable prognosis. This may be due to the effects of the infused NK cells on encountered cancer cell targets, including circulating tumor cells, as NK cells have been observed to lyse circulating tumour emboli efficiently (25). Starting NK cell-based AIET immediately after diagnosis, as in the present case, may be advantageous, as these NK cells may also act on breast cancer cells that migrate to the bone marrow during the early stages of the disease, as well as targeting treatment-resistant breast cancer stem cells $(3,5,6)$. Therefore, an assessment of NK cell expansion potential and cytotoxicity following conventional therapies in several patients may elucidate the potential loopholes of the immune system through which cancer is able to evade these immune cells, leading to disease recurrence and resistance to treatment. Further studies focusing on improving or modifying current therapeutic strategies for cancer so that they do not compromise the application of autologous immune cells must be performed, after which the approach of combining AIET with conventional therapies may be suggested for similar cases with proper validation.

In conclusion, combining NK cell-based AIET with surgery and chemotherapy was associated with $>28$ months of disease-free survival in a patient with stage IIIA IBC. The in vitro expansion potential of NK cells gradually declined with subsequent dosages of chemotherapy and markedly after surgery in this patient, which warrants an assessment of the immune system during therapies that compromise immunity to allow spread of cancer, and also to properly validate the timings of starting such immune-enhancing therapies. In addition, targeted cancer therapies that do not compromise the immune system are urgently required.

\section{Acknowledgements}

The authors would like to thank the M/S Chennai Cell Cluster (CCC) for technical advice, the Loyola ICAM College of Engineering Technology (LICET) and the Loyola Institute of Frontier Energy (LIFE) for their support.

\section{References}

1. World Cancer Report. International Agency for Research on Cancer (2008). Available at: http://www.iarc.fr/en/publications/pdfs-online/wcr/2008/index.php Accessed April 29, 2016.

2. Dittmer J and Rody A: Cancer stem cells in breast cancer. Histol Histopathol 28: 827-838, 2013.

3. Corcoran KE, Trzaska KA, Fernandes H, Bryan M, Taborga M, Srinivas V,Packman K, Patel PS and Rameshwar P: Mesenchymal stem cells in early entry of breast cancer into bone marrow. PLoS One 3: e2563, 2008.

4. Terunuma H, Deng X, Dewan Z, Fujimoto S and Yamamoto N: Potential role of NK cells in the induction of immune responses: Implications for NK cell-based immunotherapy for cancers and viral infections. Int Rev Immunol 27: 93-110, 2008.

5. Tseng HC, Arasteh A, Paranjpe A, Teruel A, Yang W, Behel A, Alva JA, Walter G, Head C, Ishikawa TO, et al: Increased lysis of stem cells but not their differentiated cells by natural killer cells; de-differentiation or reprogramming activates NK cells. PLoS One 5: e11590, 2010.

6. Yin T, Wang G, He S, Liu Q, Sun J and Wang Y: Human cancer cells with stem cell-like phenotype exhibit enhanced sensitivity to the cytotoxicity of IL-2 and IL-15 activated natural killer cells Cell Immunol 300: 41-45, 2016.

7. Takayama T, Sekine T, Makuuchi M, Yamasaki S, Kosuge T, Yamamoto J, Shimada K, Sakamoto M, Hirohashi S, Ohashi Y and Kakizoe T: Adoptive immunotherapy to lower postsurgical recurrence rates of hepatocellular carcinoma: A randomised trial. Lancet 356: 802-807, 2000.

8. Kono K, Takahashi A, Ichihara F, Amemiya H, Iizuka H, Fujii H, Sekikawa T and Matsumoto Y: Prognostic significance of adoptive immunotherapy with tumor-associated lymphocytes in patients with advanced gastric cancer: A randomized trial. Clin Cancer Res 8: 1767-1771, 2002.

9. Egawa K: Immuno-cell therapy of cancer in Japan. Anticancer Res 24: 3321-3326, 2004.

10. Takada M, Terunuma H, Deng X, Dewan MZ, Saji S, Kuroi K, Yamamoto N and Toi M: Refractory lung metastasis from breast cancer treated with multidisciplinary therapy including an immunological approach. Breast Cancer 18: 64-67, 2011.

11. Domschke C, Ge Y, Bernhardt I, Schott S, Keim S, Juenger S, Bucur M, Mayer L, Blumenstein M, Rom J, et al: Long-term survival after adoptive bone marrow $\mathrm{T}$ cell therapy of advanced metastasized breast cancer: Follow-up analysis of a clinical pilot trial. Cancer Immunol Immunother 62: 1053-1060, 2013. 
12. Kotsakis A, Sarra E, Peraki M, Koukourakis M, Apostolaki S, Souglakos J, Mavromanomakis E, Vlachonikolis J and Georgoulias V: Docetaxel-induced lymphopenia in patients with solid tumors: A prospective phenotypic analysis. Cancer 89: 1380-1386, 2000.

13. Bloom HJ and Richardson WW: Histological grading and prognosis in breast cancer; a study of 1409 cases of which 359 have been followed for 15 years. Br J Cancer 11: 359-377, 1957.

14. Sobin LH, Gospodarowicz MK and Wittekind CH: TNM Classification of Malignant Tumors. 7th edition. Wiley-Blackwell, Oxford, 2009.

15. Premkumar S, Dedeepiya VD, Terunuma H, Senthilkumar R Srinivasan T, Reena HC, Preethy S and Abraham SJ: Cell based autologous immune enhancement therapy (AIET) after radiotherapy in a locally advanced carcinoma of the cervix. Case Rep Oncol Med 2013: 903094, 2013.

16. Manjunath SR, Ramanan G, Dedeepiya VD, Terunuma H, Deng X, Baskar S, Senthilkumar R, Thamaraikannan P, Srinivasan T, Preethy S and Abraham SJ: Autologous immune enhancement therapy in recurrent ovarian cancer with metastases: A case report. Case Rep Oncol 5: 114-118, 2012.

17. Dedeepiya V, Terunuma H, Deng X, Baskar S, Manjunath S, Senthilkumar R, Murugan P, Thamaraikannan P, Srinivasan T, Preethy $\mathrm{S}$ and Abraham SJ: A comparative analysis of in vitro expansion of natural killer cells of a patient with autoimmune haemolytic anaemia and ovarian cancer with patients with other solid tumours. Oncol Lett 3: 435-440, 2012.

18. Gogia A, Raina V, Deo SV, Shukla NK, Mohanti BK and Sharma DN: Inflammatory breast cancer: A single centre analysis. Asian Pac J Cancer Prev 15: 3207-3210, 2014.
19. Sevko A, Sade-Feldman M, Kanterman J, Michels T, Falk CS, Umansky L, Ramacher M, Kato M, Schadendorf D, Baniyash M and Umansky V: Cyclophosphamide promotes chronic inflammation-dependent immunosuppression and prevents antitumor response in melanoma. J Invest Dermatol 133: 1610-1619, 2013.

20. Tsavaris N, Kosmas C, Vadiaka M, Kanelopoulos P and Boulamatsis D: Immune changes in patients with advanced breast cancer undergoing chemotherapy with taxanes. Br J Cancer 87: 21-27, 2002.

21. Tai LH, de Souza CT, Bélanger S, Ly L, Alkayyal AA, Zhang J, Rintoul JL, Ananth AA, Lam T, Breitbach CJ, et al: Preventing postoperative metastatic disease by inhibiting surgery-induced dysfunction in natural killer cells. Cancer Res 73: 97-107, 2013.

22. Fridman WH, Remark R, Goc J, Giraldo NA, Becht E, Hammond SA, Damotte D, Dieu-Nosjean $M C$ and Sautès-Fridman C: The immune microenvironment: A major player I human cancers. Int Arch Allergy Immunol 164: 13-26, 2014.

23. Sznurkowski JJ, Zawrocki A and Biernat W: Subtypes of cytotoxic lymphocytes and natural killer cells infiltrating cancer nests correlate with prognosis in patients with vulvar squamous cell carcinoma. Cancer Immunol Immunother 63: 297-303, 2014.

24. Shafer D, Smith MR, Borghaei H, Millenson MM, Li T, Litwin S, Anad R and Al-Saleem T: Low NK cell counts in peripheral blood are associated with inferior overall survival in patients with follicular lymphoma. Leuk Res 37: 1213-1235, 2013.

25. Hanna N and Fidler IJ: Role of natural killer cells in the destruction of circulating tumor emboli. J Natl Cancer Inst 65: 801-809, 1980. 